\title{
Negative feedback enables structurally signed steady-state influences in artificial biomolecular networks
}

\author{
Giulia Giordano $^{a}$ and Elisa Franco ${ }^{b}$
}

\begin{abstract}
We examine the capacity of artificial biomolecular networks to respond to perturbations with structurally signed steady-state changes. We consider network architectures designed to balance their output production as a function of downstream demand: the species producing the output, called a source, up- or down-regulates its production rate as a function of the demand. Using an exact algorithm we show that, in certain negative feedback architectures, changes in the total source concentration cause structurally signed variations of the steady-state output concentration, regardless of reaction rate parameters. Conversely, positive feedback schemes can exhibit the same signed behaviour for reasonable (but not for arbitrary) values of the parameters. Numerical simulations demonstrate how the steady-state concentrations of different network architectures vary, responding to perturbations in total source amounts, consistently with our structural previsions.
\end{abstract}

\section{INTRODUCTION}

One of the research frontiers in control theory is the development of techniques and design principles to engineer biological systems for predictable responses. Recent advances in molecular biology and nanotechnology provide modern bioengineers with a variety of molecular parts that can be modularly interconnected to build systems of ever increasing complexity. This availability of programmable parts motivates and fuels the rational design of de novo artificial networks. Yet, uncertainty in the properties of these parts and interconnections requires that parameter-independent criteria be used for performance evaluation [1], [3], [4], [12].

Biomolecular feedback architectures have been recently identified to stoichiometrically regulate the production of output components as a function of downstream demand [9]: as a case study, we considered networks where the output (R) of each network element (T) contributes to the formation of one or more complexes $(\mathrm{P})$; output not used to form product triggers feedback reactions to regulate its production, so that it is stoichiometrically matched with demand [9]. Architectures based either on negative or positive feedback have been proposed [6], [7], [9], [10]. Fig. 1a illustrates an example pathway where two network elements concur to form a product $\mathrm{P}$ and are controlled with negative autoregulation: when an output species $R_{\mathrm{i}}$ is in excess, relative to the amount effectively used for product formation, it reduces its own generation rate. This network was experimentally implemented using synthetic transcriptional systems [9], [17], [18], where $T_{i}$ are artificial genes and $R_{i}$ their RNA transcripts, which

\footnotetext{
${ }^{a}$ Dept. of Automatic Control and LCCC Linnaeus Center, Lund University, Lund, Sweden. giulia.giordanodcontrol.lth.se

${ }^{b}$ Department of Mechanical Engineering, University of California at Riverside, Riverside, CA 92521, USA. efranco@engr.ucr.edu
}

bind to form a complex P. Fig. 1b shows a two-element network with cross-activation: when a species $R_{i}$ is in excess, it increases the generation rate of species $R_{j}$. Both schemes have been scaled to the case of $n$ elements (hence, $n$ interacting $\mathrm{R}$ species) [9], [13], considering different interconnection topologies: in the single-product interconnection, all of the outputs combine into a single product (Fig. 2 A); in the neighbour interconnection, each output participates in two products (binding to its two output-neighbours in an ideal closed chain, see Fig. 2 B); in the handshake interconnection, each output participates in $n-1$ products, binding to all of the other outputs (Fig. 2 C).

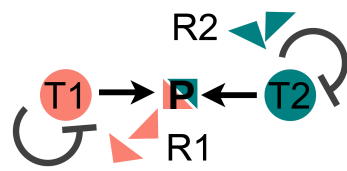

(a) Self-inhibition

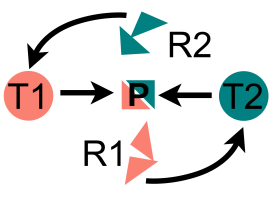

(b) Cross-activation
Fig. 1: Negative and positive rate-regulation concepts. [9]

Negative feedback networks for production rate control are expected to exhibit "tracking" properties when the total concentration of each element $\mathrm{T}$ is varied, as discussed in [12] for the case of a two-element scheme. These properties are akin to correlated behaviours that arise when a single enzyme processes several substrates: if the enzyme is overloaded, then an increase in the total concentration of one of the substrates causes an increase in the free concentration of the others, as shown in [5]. The architecture of a negative autoregulation circuit, with outputs creating a single product, is similar: several output components assemble in one product and, if they are not used up, their corresponding production source is inhibited. If the total concentration of one of the elements increases, it leads to an increase in the corresponding output. Hence, the other output species should decrease at first (there is a creation of more binding sites for product formation) and the other source element concentrations should rise, because stoichiometric self-inhibition is reduced. If an output species $R_{i}$ decreases due to the reduction in the total concentration of the corresponding source $T_{i}$, then the concentration of all other $R_{j}$ should increase, because the number of binding sites progressively decreases, and the corresponding $T_{j}$ concentrations should decrease, due to an increased self-inhibition. This "coherent propagation" of perturbations can be considered a tracking behavior, which has been experimentally shown in other negative feedback modules fueled by cooperative (rather than stoichiometric) interactions [15]. 

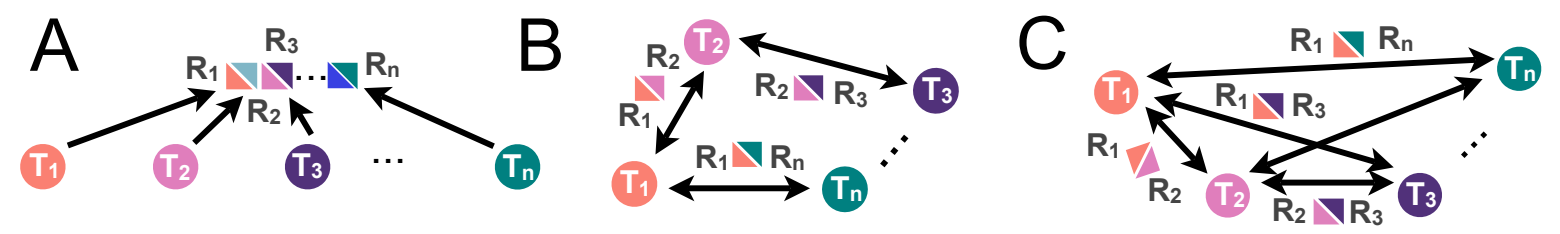

Fig. 2: Different interconnection topologies: single-product (A), neighbour (B) and handshake (C). [9]

Do these properties of steady-state input-output variations hold for positive feedback as well? For negative (or, in case, positive) feedback schemes, are these properties structural, i.e., "inherent" to circuit design and independent of the specific values of the parameters?

To answer these questions, we exploit a result relying on the implicit function theorem and the algorithms proposed in [12] to structurally compute the steady-state input-output influence based on the so-called $B D C$-decomposition [2], [4], [11], [12]. We show that, in the 2-element case, negativefeedback systems exhibit structurally signed input-output influences for all state variables, while positive-feedback systems exhibit input-output influences having a specific sign for reasonable parameter values (but not structurally, at least for some of the variables). The same phenomena occur for more than two elements: negative feedback circuits still feature structurally signed influences, for all variables in the single-product interconnection, just for some of the variables in the other topologies; positive feedback circuits can exhibit the same signed behaviour for reasonable (as in [9]) values of the parameters. Analytical results are paired with simulations performed for rate-regulatory networks of growing size, with negative and positive feedback regulation, and with different output interconnection topologies: we stimulate the system by suddenly varying a total source amount and we evaluate changes in steady state concentrations.

\section{Steady-State InPut-OutPut Influences}

The steady-state input-output influence is the ensuing variation of the steady state of a certain variable of the system (seen as the system output), upon a variation in a relevant variable or parameter (which can be seen as an input for the system). Of course, different variables of the system may respond with a steady-state variation that has the same sign as the input variation, the opposite sign, or is zero. The steadystate input-output influence is structurally signed if it always has the same sign (positive, negative, or zero), regardless of the choice of parameter values. [12]

To assess the steady-state input-output influence in a generic nonlinear system

$$
\begin{aligned}
& \dot{x}(t)=f(x(t), u(t)), \\
& y(t)=g(x(t)),
\end{aligned}
$$

where $f$ and $g$ are continuously differentiable, $x \in \mathbb{R}^{n}$, $u$ is a scalar input and $y$ a scalar output, we assume that there exists an asymptotically stable equilibrium point $\bar{x}$, corresponding to $\bar{u}$, such that $f(\bar{x}, \bar{u})=0$. Then, both the state asymptotic value $\bar{x}(u)$ and the output asymptotic value $\bar{y}(u)=g(\bar{x})$ are functions of $u$. If the considered input variation is small enough to ensure that asymptotic stability of $\bar{x}(u)$ is preserved (being the eigenvalues of the Jacobian matrix continuously dependent on the entries, which are in turn continuous functions of $u$ ), then the implicit function theorem provides an analytic expression for the derivative of the steady-state input-output map that relates $y$ to $u$ :

$$
\frac{\partial \bar{y}}{\partial \bar{u}}=\left.\left.\frac{\partial g}{\partial x}\right|_{\bar{x}}\left(-\left.\frac{\partial f}{\partial x}\right|_{(\bar{x}, \bar{u})}\right)^{-1} \frac{\partial f}{\partial u}\right|_{(\bar{x}, \bar{u})} .
$$

Given the linearization of the system in a neighborhood of $\bar{x}$, with $z(t)=x(t)-\bar{x}, v(t)=u(t)-\bar{u}, w(t)=y(t)-\bar{y}$,

$$
\begin{aligned}
& \dot{z}(t)=A z(t)+B v(t), \\
& w(t)=H z(t),
\end{aligned}
$$

where $A_{i j}=\left.\frac{\partial f_{i}}{\partial x_{j}}\right|_{(\bar{x}, \bar{u})}, B_{i}=\left.\frac{\partial f_{i}}{\partial u}\right|_{(\bar{x}, \bar{u})}$ and $C_{i}=\left.\frac{\partial g}{\partial x_{i}}\right|_{\bar{x}}$, the input-output influence can be expressed as [12]

$$
\frac{\partial \bar{y}}{\partial \bar{u}}=C(-A)^{-1} B=\frac{n(0)}{d(0)}
$$

where $d(0)=\operatorname{det}(-A)>0$, in view of stability, while

$$
n(0)=\operatorname{det}\left[\begin{array}{rr}
-A & -B \\
C & 0
\end{array}\right] .
$$

Note that $n(0)$ and $d(0)$ are the numerator and the denominator of the system transfer function $F(s)=n(s) / d(s)=$ $C(s I-A)^{-1} B$, computed at $s=0$.

The above expression can be used to evaluate input-output influences for a given choice of parameters. To evaluate the structural (parameter-free) input-output influence, [12] proposes an efficient algorithm, yielding a "+" if increasing the input structurally results in an increase in the steadystate value of the considered variable, a "-" if it structurally results in a decrease, a " 0 " if the steady-state of the considered variable is unchanged, and a "?" if the behaviour is parameter-dependent. The algorithm in [12] is applicable to systems admitting a particular type of decomposition, called $B D C$-decomposition [2], [4], [11], [12]. This category includes systems with a signed Jacobian as a particular case, because the $B D C$-decomposition relies on the fact that, for this class of systems, the determinant in (1) can be written as a multiaffine function of suitable positive parameters, related to the system partial derivatives (see [11], [12] for details). As shown in the next section, rate-regulatory models have indeed a sign-definite Jacobian, hence the algorithm in [12] can be applied to structurally evaluate the sign of steady-state input-output influences.

\section{Analysis of Production Control Networks}

We consider molecular networks where each element $i$ is composed of a molecular production source $T_{i}$ and the 
corresponding output species $\mathrm{R}_{\mathrm{i}}$. A source can be active $\left(\mathrm{T}_{\mathrm{i}}\right)$ or inactive $\left(\mathrm{T}_{\mathrm{i}}^{*}\right)$, and its total concentration is conserved $\left[\mathrm{T}_{\mathrm{i}}\right]+\left[\mathrm{T}_{\mathrm{i}}^{*}\right]=\left[\mathrm{T}_{\mathrm{i}}^{\mathrm{tot}}\right]$. Active source produces $\mathrm{R}_{\mathrm{i}}$ according to the linear reaction $\mathrm{T}_{\mathrm{i}} \stackrel{\beta}{\rightarrow} \mathrm{R}_{\mathrm{i}}+\mathrm{T}_{\mathrm{i}}$; then, the produced $\mathrm{R}_{\mathrm{i}}$ can interact with other output species, reacting at rate $k$. Outputs either inactivate their own production source $\left(\mathrm{R}_{\mathrm{i}}+\mathrm{T}_{\mathrm{i}} \stackrel{\delta}{\rightarrow} \mathrm{T}_{\mathrm{i}}^{*}\right.$, negative feedback regulation), or enable the activation of other sources $\left(\mathrm{R}_{\mathrm{i}}+\mathrm{T}_{\mathrm{j}}^{*} \stackrel{\delta}{\rightarrow} \mathrm{T}_{\mathrm{j}}\right.$, positive feedback regulation). Sources spontaneously revert to an active state $\mathrm{T}_{\mathrm{i}}^{*} \stackrel{\alpha}{\rightarrow} \mathrm{T}_{\mathrm{i}}$ (in negative feedback architectures) or to an inactive state $\mathrm{T}_{\mathrm{i}} \stackrel{\alpha}{\rightarrow} \mathrm{T}_{\mathrm{i}}^{*}$ (positive feedback architectures).

In the following, we consider a specific kind of molecular implementation of these architectures using transcriptional systems [6], [7], [9], [10]. Production sources T are synthetic gene templates, which are inactive when the promoter (the binding region of RNA polymerase, RNAP) is incomplete, or active when the promoter is complete. Promoter activation/inactivation is done via strand displacement [17], and RNA species $\mathrm{R}$ transcribed by the templates are designed to either interact to form a product or to inactivate/activate templates. In these systems, enzyme RNase $\mathrm{H}$ allows gene reactivation or inactivation following RNA regulatory activity. In general, other implementations are possible where regulators operate stoichiometrically, such as using TALENs or CRISPR [16], [19]. We now consider architectures based on both negative and positive regulatory loops, to (structurally) assess steady-state input-output influences.

\section{A. Negative-Feedback Schemes}

A 2-gene rate-regulatory feedback circuit based on selfrepression is described by the reactions [6], [7], [9]

$$
\mathrm{T}_{\mathrm{i}}^{*} \stackrel{\alpha_{i}}{\rightarrow} \mathrm{T}_{\mathrm{i}}, \quad \mathrm{T}_{\mathrm{i}} \stackrel{\beta_{i}}{\rightarrow} \mathrm{R}_{\mathrm{i}}+\mathrm{T}_{\mathrm{i}}, \quad \mathrm{R}_{\mathrm{i}}+\mathrm{T}_{\mathrm{i}} \stackrel{\delta_{i}}{\rightarrow} \mathrm{T}_{\mathrm{i}}^{*}, \quad \mathrm{R}_{1}+\mathrm{R}_{2} \stackrel{k}{\rightarrow} \mathrm{P},
$$

where $i \in\{1,2\}$ (see Fig. 1a). We also consider a spontaneous RNA degradation $\mathrm{R}_{\mathrm{i}} \stackrel{\phi_{i}}{\rightarrow} \emptyset$. Using mass action kinetics, and denoting $x_{1}=\left[\mathrm{T}_{1}\right], x_{2}=\left[\mathrm{R}_{1}\right], x_{3}=\left[\mathrm{T}_{2}\right], x_{4}=\left[\mathrm{R}_{2}\right]$, the reactions can be associated with the ODE system:

$$
\left\{\begin{array}{l}
\dot{x}_{1}=\alpha_{1}\left(x_{1}^{t o t}-x_{1}\right)-\delta_{1} x_{1} x_{2} \\
\dot{x}_{2}=\beta_{1} x_{1}-k x_{2} x_{4}-\delta_{1} x_{1} x_{2}-\phi_{1} x_{2} \\
\dot{x}_{3}=\alpha_{2}\left(x_{3}^{t o t}-x_{3}\right)-\delta_{2} x_{3} x_{4} \\
\dot{x}_{4}=\beta_{2} x_{3}-k x_{2} x_{4}-\delta_{2} x_{3} x_{4}-\phi_{2} x_{4}
\end{array}\right.
$$

Positivity of the system is expected (the variables physically represent concentrations, hence, nonnegative quantities); moreover, it can be proved that the trajectories are globally asymptotically bounded in the set $\mathcal{B}_{n}=\left\{x \in \mathbb{R}^{4}\right.$ : $\left.0 \leq x_{1} \leq x_{1}^{t o t}, 0 \leq x_{2} \leq x_{2}^{+}, 0 \leq x_{3} \leq x_{3}^{t o t}, 0 \leq x_{4} \leq x_{4}^{+}\right\}$, where $x_{2}^{+}=\frac{\beta_{1}}{\phi_{1}} x_{1}^{t o t}$ and $x_{4}^{+}=\frac{\beta_{2}}{\phi_{2}} x_{2}^{t o t}$.

Proposition 1: System (2) is positive and its solutions are globally asymptotically bounded in $\mathcal{B}_{n}$ for any nonnegative initial condition such that $x_{1}(0) \leq x_{1}^{\text {tot }}$ and $x_{3}(0) \leq x_{3}^{\text {tot }}$.

Proof: The system positivity is immediately proved by noticing that, when $x_{i}=0$, the corresponding equation $\dot{x}_{i}$ has only nonnegative terms in the right-hand side (hence, $x_{i}$ cannot decrease any longer). The bounds $x_{1}(t) \leq x_{1}^{t o t}$ and $x_{3}(t) \leq x_{3}^{t o t}$ for all $t$ immediately descend from the first and third equations in (2). The conditions $0 \leq x_{2}(t) \leq x_{2}^{+}$and $0 \leq x_{4}(t) \leq x_{4}^{+}$are satisfied for all $t>0$, as long as they hold for $t=0$; also, they are asymptotically satisfied for all initial conditions. In fact, since $\dot{x}_{2} \leq \beta_{1} x_{1}^{\text {tot }}-\phi_{1} x_{2}$, based on the comparison principle, $x_{2}(t) \leq x_{2}^{+}+\left[x_{2}(0)-x_{2}^{+}\right] e^{-\phi_{1} t}$, and analogously $x_{4}(t) \leq x_{4}^{+}+\left[x_{4}(0)-x_{4}^{+}\right] e^{-\phi_{1} t}$.

Boundedness implies the existence of an equilibrium point $\bar{x}$ inside $\mathcal{B}_{n}$ ([20], [21], [22]). The Jacobian matrix $A_{n e g}$, corresponding to the linearization of the system around $\bar{x}$, is shown in Table I. Since at steady state $\left(\beta_{1}-\delta_{1} \bar{x}_{2}\right) \bar{x}_{1}-$ $k \bar{x}_{2} \bar{x}_{4}=0$, it must be $\beta_{1}-\delta_{1} \bar{x}_{2}>0$; likewise, $\beta_{2}-\delta_{2} \bar{x}_{4}>$ 0 , hence the system Jacobian is a sign-definite matrix. Note that $\operatorname{det}\left(-A_{n e g}\right)>0$ (actually, all of the coefficients of the characteristic polynomial are positive) independent of parameter values; since the system trajectories are bounded, due to degree theory arguments [14], structural positivity of the determinant implies that the equilibrium is unique. Let us assume that such an equilibrium is asymptotically stable.

If the total gene template amount $\left[\mathrm{T}_{1}^{\mathrm{tot}}\right]$ increases, then, at steady-state, the active gene template amount $\left[\mathrm{T}_{1}\right]$ increases and therefore so does the RNA species amount $\left[\mathrm{R}_{1}\right]$. Due to the increase in $\left[R_{1}\right]$, more binding sites are created, hence the other RNA species amount $\left[\mathrm{R}_{2}\right]$ decreases, being more required and consumed in the product generation. Then, the other gene template amount $\left[\mathrm{T}_{2}\right]$ increases, because stoichiometric self-inhibition is reduced. The increase in $\left[\mathrm{T}_{2}\right]$ might in turn increase $\left[R_{2}\right]$, whose behaviour is not clearly predictable. However, the net effect is that $\left[R_{2}\right]$ decreases, as shown in [12]. (The reasoning is naturally the same if subscripts 1 and 2 are swapped.) Of course, the concentration increases provided that it can: since the reagents bind according to a given stoichiometry, the expected increase can only occur if the reagent with the lowest concentration is augmented until it is no more a bottleneck for the output production. To analytically assess steady-state influences, we check how an increase in $x_{1}^{t o t}$ (and in $x_{3}^{t o t}$ ) influences each variable by means of the steady state derivative. This means considering the sign of the determinant in Eq. (1), by choosing $B=\left[\begin{array}{llll}1 & 0 & 0 & 0\end{array}\right]^{\top}$ when an increase in $x_{1}^{\text {tot }}$ is considered, $B=\left[\begin{array}{llll}0 & 0 & 1 & 0\end{array}\right]^{\top}$ when an increase in $x_{3}^{\text {tot }}$ is considered, and by choosing $C$ as the row vector whose entries are all zero, except for the $i$ th, which is 1 , when assessing the influence on variable $x_{i}$.

As discussed in [12], based on the tools proposed therein we obtain that, when the total concentration of a gene template varies, all of the steady-state influences are structurally signed, independent of parameter values:

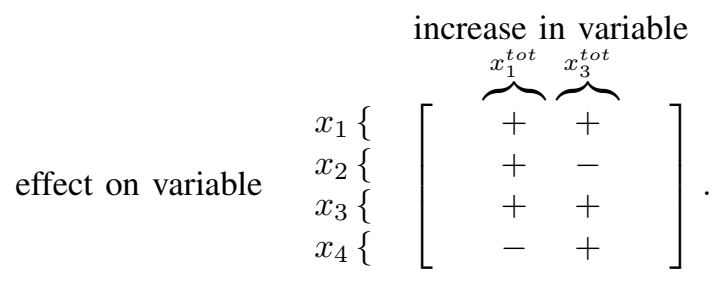

The behaviour is symmetric, as expected. For a physically reasonable choice of the parameters (consistent with [9]), the 
system evolution when $x_{1}^{\text {tot }}$ is suddenly increased is shown in Fig. 3a, exhibiting exactly the expected behaviour.

The negative auto-regulated system can be extended to the general case of $n$ genelets [9], [13], according to the different interconnection topologies in Fig. 2. Still, an increase in the total concentration of any of the gene templates, $\left[\mathrm{T}_{\mathrm{i}}^{\mathrm{tot}}\right]$, increases $\left[\mathrm{T}_{\mathrm{i}}\right]$, hence $\left[\mathrm{R}_{\mathrm{i}}\right]$; then, all the other RNAs forming products with $\mathrm{R}_{\mathrm{i}}$ should decrease at first, leading to an increase in the concentration of all other active genes (there is a creation of more binding sites due to the increase in $\left[\mathrm{R}_{\mathrm{i}}\right]$, hence stoichiometric self-inhibition is reduced). On the other hand, if $\left[\mathrm{R}_{\mathrm{i}}\right]$ decreases, due to a decrease in $\left[\mathrm{T}_{\mathrm{i}}^{\mathrm{tot}}\right]$, then all the other gene template concentrations should decrease because the number of binding sites progressively decreases, resulting in an increased self-inhibition.

The systems are described by the equations $(i=1, \ldots, n)$

$$
\left\{\begin{array}{l}
\dot{x}_{2 i-1}=\alpha_{i}\left(x_{2 i-1}^{t o t}-x_{2 i-1}\right)-\delta_{i} x_{2 i-1} x_{2 i}, \text { (templates) } \\
\dot{x}_{2 i}=\beta_{i} x_{2 i-1}-\delta_{i} x_{2 i-1} x_{2 i}-\phi_{i} x_{2 i}-P_{i},(\text { RNAs })
\end{array}\right.
$$

where

- $P_{i}=k \prod_{j=1}^{n} x_{2 j}$ for the single-product case (Fig. 2 A);

- $P_{i}=k_{2 i, 2(i-1)} x_{2 i} x_{2(i-1)}+k_{2 i, 2(i+1)} x_{2 i} x_{2(i+1)}$ (with $i-1=n$ if $i=1$ and $i+1=1$ if $i=n$ ) for the neighbour connection (Fig. 2 B);

- $P_{i}=\sum_{j \neq i} k_{2 i, 2 j} x_{2 i} x_{2 j}$ for the handshake connection (Fig. $2 \mathrm{C}$ ).

Positivity and boundedness can still be proved, as for the 2 -gene system. Also, the determinant is still structurally positive, thus ensuring uniqueness of the equilibrium. In the case of a single-product interconnection (for instance, with three genes), the influence is structurally determined:

effect on variable

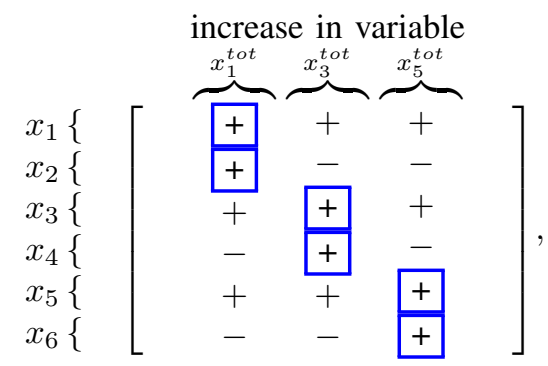

consistently with the simulations shown in Fig. 3b. In the case of a 3-gene neighbour/handshake interconnection, instead, only the influences squared in blue in the above sign matrix are structurally determined; the others, however, despite being "?" from a structural point of view, still have the sign provided above for reasonable choices of the parameters, as is shown by the simulation in Fig. 3c.

The results can be extended for more than 3 genes: for different interconnection topologies, the simulated system behaviour is shown in Figs. 3d-e-f. Note that, for the neighbour connection, the increase in $x_{1}^{\text {tot }}$ induces the increase in $x_{2}$ ( $\left[\mathrm{R}_{1}\right]$, the corresponding RNA species), but not the decrease of all of the other RNA species: in fact $x_{6}$ (the concentration of $\mathrm{R}_{3}$, which in this interconnection topology is not forming products with $\mathrm{R}_{1}$ ) increases as well, due to the decrease in $x_{4}$ and $x_{8}$ (the concentrations of $\mathrm{R}_{2}$ and $\mathrm{R}_{4}$, with which $\mathrm{R}_{1}$ is forming products); and also $x_{5}$ decreases (due to the increase in $x_{6}$, which amplifies the self-inhibition effect), while other gene template concentrations increase. In the handshake and single-product interconnections, instead, all of the other RNA species are binding with $\mathrm{R}_{1}$, hence all of their concentrations decrease.

\section{B. Positive-Feedback Schemes}

The 2-gene rate-regulatory feedback circuit based on cross-activation is described by the reactions [6], [9], [10]

$$
\mathrm{T}_{\mathrm{i}} \stackrel{\alpha_{i}}{\rightarrow} \mathrm{T}_{\mathrm{i}}^{*}, \quad \mathrm{~T}_{\mathrm{i}} \stackrel{\beta_{i}}{\rightarrow} \mathrm{R}_{\mathrm{i}}+\mathrm{T}_{\mathrm{i}}, \quad \mathrm{R}_{\mathrm{i}}+\mathrm{T}_{\mathrm{j}}^{*} \stackrel{\delta_{i j}}{\rightarrow} \mathrm{T}_{\mathrm{j}}, \quad \mathrm{R}_{1}+\mathrm{R}_{2} \stackrel{k}{\rightarrow} \mathrm{P},
$$

where $i \in\{1,2\}$ (see Fig. 1b). We also consider a spontaneous RNA degradation $\mathrm{R}_{\mathrm{i}} \stackrel{\phi_{i}}{\rightarrow} \emptyset$. Using mass action kinetics, and denoting $x_{1}=\left[\mathrm{T}_{1}\right], x_{2}=\left[\mathrm{R}_{1}\right], x_{3}=\left[\mathrm{T}_{2}\right], x_{4}=\left[\mathrm{R}_{2}\right]$, the reactions can be associated with the ODE system:

$$
\left\{\begin{array}{l}
\dot{x}_{1}=-\alpha_{1} x_{1}+\delta_{14} x_{4}\left(x_{1}^{t o t}-x_{1}\right) \\
\dot{x}_{2}=\beta_{1} x_{1}-k x_{2} x_{4}-\delta_{23} x_{2}\left(x_{3}^{t o t}-x_{3}\right)-\phi_{1} x_{2} \\
\dot{x}_{3}=-\alpha_{2} x_{3}+\delta_{23} x_{2}\left(x_{3}^{t o t}-x_{3}\right) \\
\dot{x}_{4}=\beta_{2} x_{3}-k x_{2} x_{4}-\delta_{14} x_{4}\left(x_{1}^{t o t}-x_{1}\right)-\phi_{2} x_{4}
\end{array}\right.
$$

Given the set $\mathcal{B}_{p}=\left\{x \in \mathbb{R}^{4}: 0 \leq x_{1} \leq x_{1}^{\text {tot }}, 0 \leq x_{2} \leq\right.$ $\left.x_{2}^{+}, 0 \leq x_{3} \leq x_{3}^{\text {tot }}, 0 \leq x_{4} \leq x_{4}^{+}\right\}$, where $x_{2}^{+}=\frac{\beta_{1}}{\phi_{1}} x_{1}^{t o t}$ and $x_{4}^{+}=\frac{\beta_{2}}{\phi_{2}} x_{2}^{t o t}$, the following result can be proved along the lines of Proposition 1.

Proposition 2: System (3) is positive and its solutions are globally asymptotically bounded in $\mathcal{B}_{p}$ for any nonnegative initial condition such that $x_{1}(0) \leq x_{1}^{\text {tot }}$ and $x_{3}(0) \leq x_{3}^{\text {tot }}$.

Hence, an equilibrium point $\bar{x}$ exists inside $\mathcal{B}_{p}$ ([20], [21], [22]); the Jacobian matrix $A_{p o s}$, corresponding to the linearization of the system around $\bar{x}$, is shown in Table II. The system Jacobian is sign-definite. Even though $\operatorname{det}\left(-A_{\text {pos }}\right)$ is not structurally positive, it is positive for a reasonable choice of the parameters (due to the order of magnitude of the parameters in the real system, the only term that can be negative is much smaller). Assuming that the equilibrium is asymptotically stable, the computation of the input-output influences according to the methods in [12] provides:

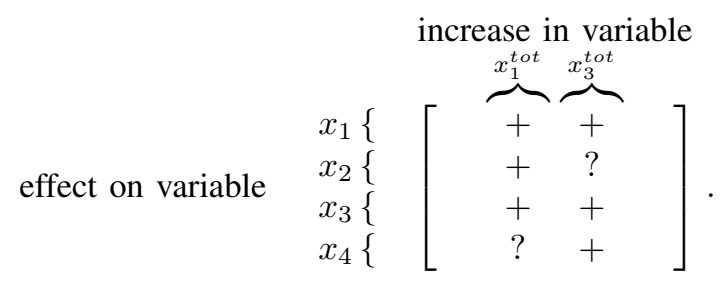

Therefore, when the gene template concentration is varied, steady-state input-output influences are structurally signed for most of the species concentrations, but not for all of them. However, both of the indeterminate expressions are actually negative for reasonable choices of the parameters (as in [9]); hence, if $x_{1}^{\text {tot }}$ increases, $x_{4}$ decreases and, if $x_{3}^{\text {tot }}$ increases, $x_{2}$ decreases as well, consistently with the simulations in Fig. 4a. Therefore, for suitable parameter values, the behaviour in the case of positive feedback (crossactivation) seems to be the same as in the case of negative 


$$
A_{n e g}=\left[\begin{array}{cccc}
-\left(\alpha_{1}+\delta_{1} \bar{x}_{2}\right) & -\delta_{1} \bar{x}_{1} & 0 & 0 \\
\beta_{1}-\delta_{1} \bar{x}_{2} & -\left(\delta_{1} \bar{x}_{1}+k \bar{x}_{4}+\phi_{1}\right) & 0 & -k \bar{x}_{2} \\
0 & 0 & -\left(\alpha_{2}+\delta_{2} \bar{x}_{4}\right) & -\delta_{2} \bar{x}_{3} \\
0 & -k \bar{x}_{4} & \beta_{2}-\delta_{2} \bar{x}_{4} & -\left(\delta_{2} \bar{x}_{3}+k \bar{x}_{2}+\phi_{2}\right)
\end{array}\right]
$$

TABLE I: Jacobian of the 2-gene system with negative auto-regulation.

feedback (self-inhibition), but in the positive feedback case the property cannot be always ensured by means of structural arguments: we have to rely on our knowledge of actual experimental parameters (at least, of their order of magnitude).

Also the cross-activation regulatory scheme can be adapted to the general case of $n$ genelets [9], [13], with various interconnection topologies (see Fig. 2). The system becomes:

$$
\left\{\begin{array}{l}
\dot{x}_{2 i-1}=-\alpha_{i} x_{2 i-1}+F_{2 i-1},(\text { templates }) \\
\dot{x}_{2 i}=\beta_{i} x_{2 i-1}-F_{2 i}-\phi_{i} x_{2 i}-P_{i}, \text { (RNAs) }
\end{array}\right.
$$

with $i=1, \ldots, n$ and

- $F_{2 i-1}=\delta_{i}\left(x_{2 i-1}^{t o t}-x_{2 i-1}\right) \prod_{j \neq i} x_{2 j}$, $F_{2 i}=\delta_{i} x_{2 i} \prod_{j \neq i}\left(x_{2 j-1}^{t o t}-x_{2 j-1}\right)$, $P_{i}=k \prod_{j=1}^{n} x_{2 j}$ for the single-product connection;

- $F_{2 i-1}=\sum_{j=i \pm 1} \delta_{i j} x_{2 j}\left(x_{2 i-1}^{t o t}-x_{2 i-1}\right)$, $F_{2 i}=\sum_{j=i \pm 1} \delta_{j i} x_{2 i}\left(x_{2 j-1}^{\text {tot }}-x_{2 j-1}\right)$, $P_{i}=\sum_{j=i \pm 1} k_{i j} x_{2 i} x_{2 j}$ for the neighbour connection;

- $F_{2 i-1}=\sum_{j \neq i} \delta_{i j} x_{2 j}\left(x_{2 i-1}^{t o t}-x_{2 i-1}\right)$, $F_{2 i}=\sum_{j \neq i} \delta_{j i} x_{2 i}\left(x_{2 j-1}^{t o t}-x_{2 j-1}\right)$, $P_{i}=\sum_{j \neq i} k_{i j} x_{2 i} x_{2 j}$ for the handshake connection.

Positivity and boundedness can still be proved, as for the 2-gene system; and, again, the determinant of the resulting Jacobian is not sign-definite. However, when $n>2$, for all of the interconnection topologies with positive regulation, none of the influences is structurally determined. If the influences are computed for parameter values in the reasonable range for the real system (used in our simulations and in [9]), again, the same signs as in the negative-feedback case are obtained: this is consistent with the simulations shown in Fig. 4b-c.

\section{CONCLUDING REMARKS}

We have demonstrated that a class of demand-adaptive molecular architectures respond to certain perturbations with signed steady-state changes. Specifically, networks regulated via negative feedback exhibit structurally (i.e., for arbitrary values of reaction rates) signed steady-state variations in response to perturbations in the total concentrations of certain components. Positive feedback networks exhibit similar behaviours, which however are not structural. For our analysis, we use an exact algorithm to compute the structural influence matrix [12] of a system; the behaviour of numerically integrated solutions is consistent with our results. Our case study suggests that negative feedback architectures may be in general more "robust" than positive feedback architectures in terms of steady-state responses, where "robustness" here is intended as the occurrence of a consistent network behaviour for arbitrary choices of the reaction rates. A systematic analysis and comparison of non-trivial negative and positive feedback regulatory networks is possible using the same approach described in this manuscript, because the algorithm in [12] can easily handle large systems.

\section{ACKNOWLEDGEMENTS}

The authors thank Franco Blanchini for valuable discussions and advice. G.G. acknowledges support from the Swedish Research Council through the LCCC Linnaeus Center and the eLLIIT Excellence Center at Lund University.

\section{REFERENCES}

[1] F. Blanchini and E. Franco, "Structurally robust biological networks," BMC Systems Biology, vol. 5, no. 1, p. 74, 2011.

[2] F. Blanchini, E. Franco, and G. Giordano, "Determining the structural properties of a class of biological models," in Proc. IEEE Conf. Decis. Control, Maui (HI), USA, 2012, pp. 5505-5510.

[3] F. Blanchini, E. Franco, and G. Giordano, "A structural classification of candidate oscillatory and multistationary biochemical systems," Bull. of Mathematical Biology, vol. 76, no. 10, pp. 2542-2569, 2014.

[4] F. Blanchini and G. Giordano, "Piecewise-linear Lyapunov functions for structural stability of biochemical networks," Automatica, vol. 50 , no. 10, pp. 2482-2493, 2014.

[5] N. A. Cookson et al, "Queueing up for enzymatic processing: correlated signaling through coupled degradation," Molecular Systems Biology, vol. 7, pp. 561, 2011.

[6] E. Franco, "Analysis, design, and in vitro implementation of robust biochemical networks," Ph.D. dissertation, California Institute of Technology, 2011.

[7] E. Franco, P.-O. Forsberg, and R. M. Murray, "Design, modeling and synthesis of an in vitro transcription rate regulatory circuit," in Proc. Am. Control Conf., 2008.

[8] E. Franco, E. Friedrichs, J. Kim, R. Jungmann, R. Murray, E. Winfree, and F. C. Simmel, "Timing molecular motion and production with a synthetic transcriptional clock," Proc. of the National Academy of Sciences, vol. 108, no. 40, pp. E784-E793, 2011.

[9] E. Franco, G. Giordano, P.-O. Forsberg, and R. M. Murray, "Negative autoregulation matches production and demand in synthetic transcriptional networks," ACS Synthetic Biology, vol. 3, pp. 589-599, 2014.

[10] E. Franco and R. M. Murray, "Design and performance of in vitro transcription rate regulatory circuits," in Proc. IEEE Conf. Decis. Control, 2008.

[11] G. Giordano, "Structural analysis and control of dynamical networks," Ph.D. dissertation, Università degli Studi di Udine, 2016.

[12] G. Giordano, C. Cuba Samaniego, E. Franco, and F. Blanchini, "Computing the structural influence matrix for biological systems," Journal of Mathematical Biology, 2015.

[13] G. Giordano, E. Franco, and R. M. Murray, "Feedback architectures to regulate flux of components in artificial gene networks," in Proc. Am. Control Conf., Washington (DC), USA, 2013, pp. 4747-4752.

[14] J. Hofbauer, "An index theorem for dissipative semiflows," Rocky Mountain Journal of Math., vol. 20, no. 4, pp. 1017-1031, 1990.

[15] V. Hsiao, E. L. C. de los Santos, W. R. Whitaker, J. E. Dueber, and R. M. Murray, "Design and implementation of a biomolecular concentration tracker," ACS Syn. Bio., vol. 4, no. 2, pp. 150-161, 2015.

[16] P. D. Hsu, E. S. Lander and F. Zhang, "Development and applications of CRISPR-Cas9 for genome engineering," Cell, vol. 157, no. 6, pp. 1262-1278, 2014.

[17] J. Kim, K. S. White, and E. Winfree, "Construction of an in vitro bistable circuit from synthetic transcriptional switches," Molecular Systems Biology, vol. 2, p. 68, 2006.

[18] J. Kim and E. Winfree, "Synthetic in vitro transcriptional oscillators," Molecular Systems Biology, vol. 7, p. 465, 2011.

[19] T. Lebar et al, "A bistable genetic switch based on designable DNAbinding domain," Nature Communications, vol. 5, p. 5007, 2014.

[20] D. Richeson and J. Wiseman, "A fixed point theorem for bounded dynamical systems," Illinois Journal of Mathematics, vol. 46, no. 2, pp. 491-495, 2002.

[21] D. Richeson and J. Wiseman, “Addendum to: [20]," Illinois Journal of Mathematics, vol. 48, no. 3, pp. 1079-1080, 2004.

[22] R. Srzednicki, "On rest points of dynamical systems," Fundamento Mathematicae, vol. 126, no. 1, pp. 69-81, 1985. 


$$
A_{\text {pos }}=\left[\begin{array}{cccc}
-\left(\alpha_{1}+\delta_{14} \bar{x}_{4}\right) & 0 & 0 & \delta_{14}\left(x_{1}^{\text {tot }}-\bar{x}_{1}\right) \\
\beta_{1} & -\left[k \bar{x}_{4}+\delta_{23}\left(x_{3}^{t o t}-\bar{x}_{3}\right)+\phi_{1}\right] & \delta_{23} \bar{x}_{2} & -k \bar{x}_{2} \\
0 & \delta_{23}\left(x_{3}^{t o t}-\bar{x}_{3}\right) & -\left(\alpha_{2}+\delta_{23} \bar{x}_{2}\right) & 0 \\
\delta_{14} \bar{x}_{4} & -k \bar{x}_{4} & \beta_{2} & -\left[\delta_{14}\left(x_{1}^{t o t}-\bar{x}_{1}\right)+k \bar{x}_{2}+\phi_{2}\right]
\end{array}\right]
$$

TABLE II: Jacobian of the 2-gene system with cross-activation.
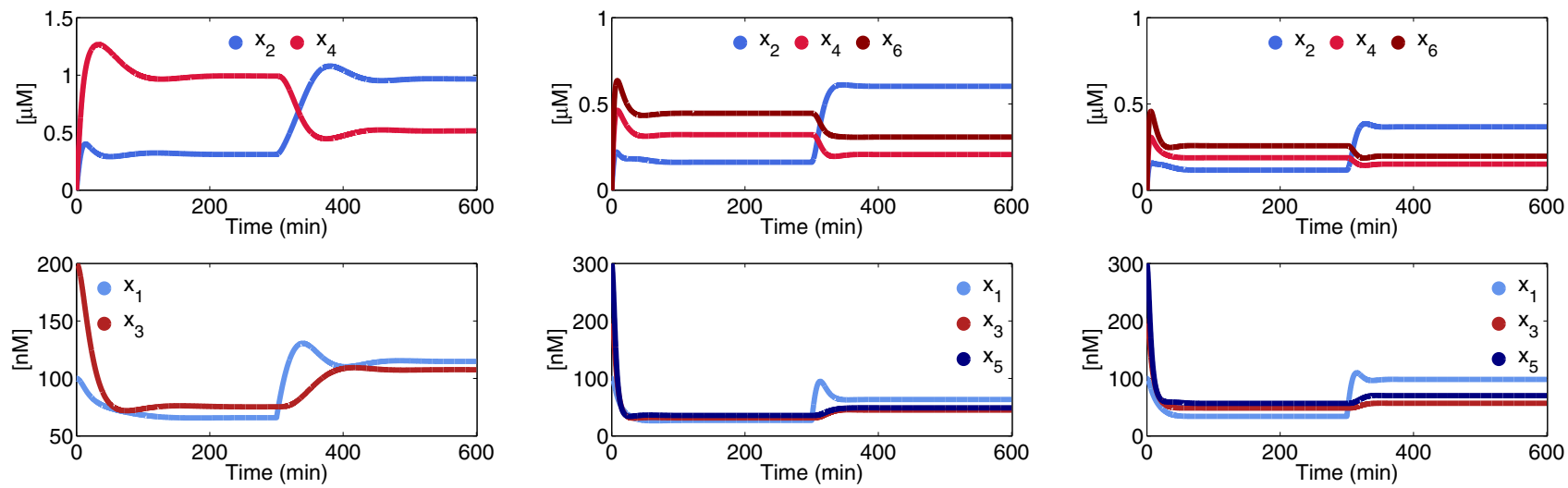

(a) 2 genes

(b) 3 genes, single-product

(c) 3 genes, neighbour/handshake
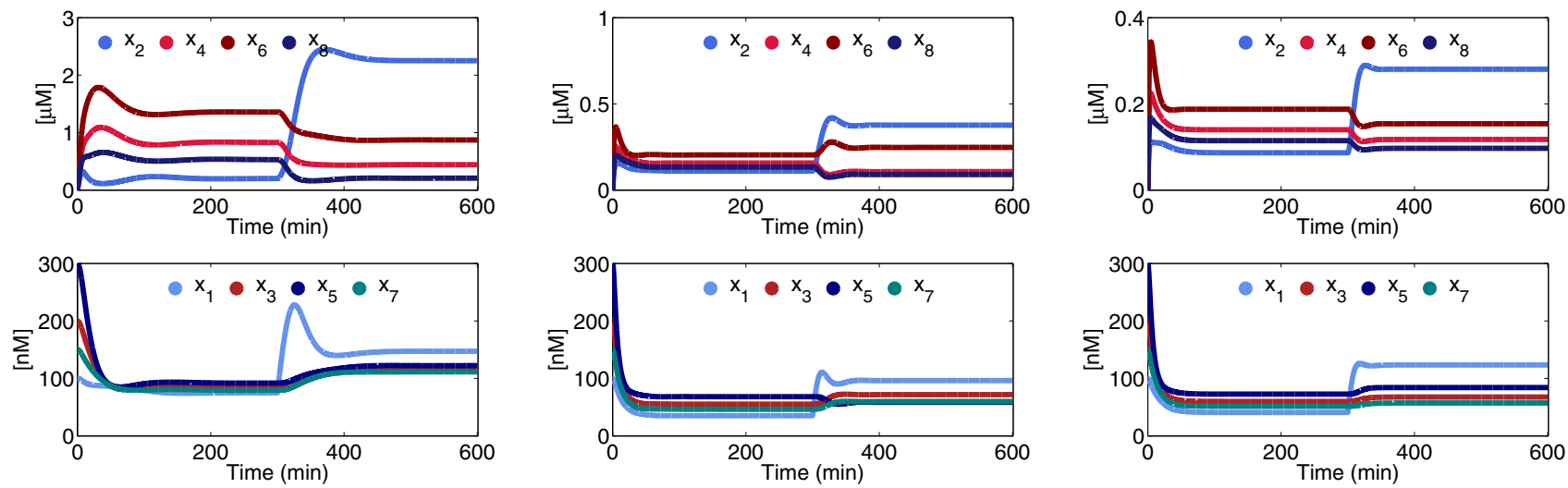

(d) 4 genes, single-product

(e) 4 genes, neighbour

(f) 4 genes, handshake

Fig. 3: Negative-feedback scheme, simulations: system evolution when $x_{1}^{\text {tot }}$ is suddenly increased from $100 \mathrm{nM}$ to $300 \mathrm{nM}$ at time $t=300 \mathrm{~min}$. The other parameters are: $\alpha_{i}=3 \cdot 10^{-4} / \mathrm{s}, \beta_{i}=1 \cdot 10^{-2} / \mathrm{s}, \delta_{i}=5 \cdot 10^{3} / \mathrm{M} / \mathrm{s}, \phi_{i}=1 \cdot 10^{-4} / \mathrm{s}, x_{2}^{\text {tot }}=200 \mathrm{nM}, x_{3}^{\text {tot }}=300 \mathrm{nM}, x_{4}^{\text {tot }}=150 \mathrm{nM}$; production rates ( $k$ ) vary from $2 \cdot 10^{3} / \mathrm{M} / \mathrm{s}$ to $6 \cdot 10^{15} / \mathrm{M} / \mathrm{s}$, depending on the interconnection topology (they need to be higher in single-product schemes).
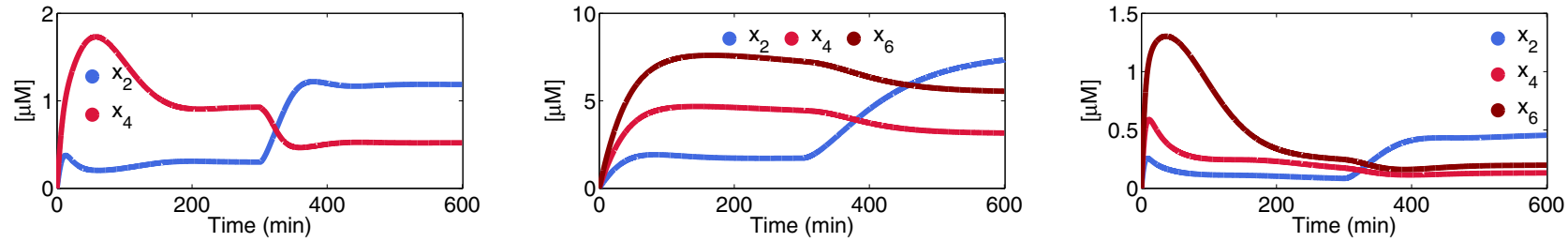

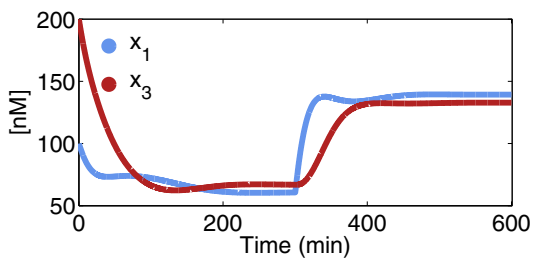

(a) 2 genes

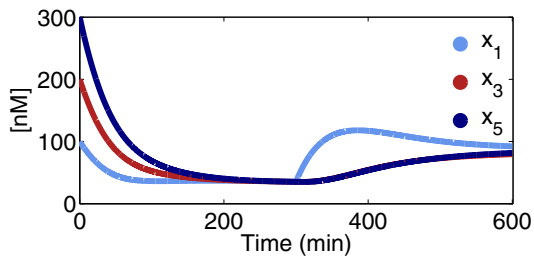

(b) 3 genes, single-product

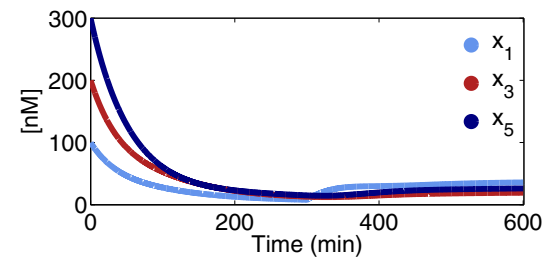

(c) 3 genes, neighbour/handshake

Fig. 4: Positive-feedback scheme, simulations: system evolution when $x_{1}^{\text {tot }}$ is suddenly increased from 100nM to $300 \mathrm{nM}$ at time $t=300 \mathrm{~min}$. The other parameters are: $\alpha_{i}=3 \cdot 10^{-4} / \mathrm{s}, \beta_{i}=1 \cdot 10^{-2} / \mathrm{s}, \phi_{i}=1 \cdot 10^{-4} / \mathrm{s}, x_{2}^{t o t}=200 \mathrm{nM}, x_{3}^{t o t}=300 \mathrm{nM}$; feedback rates are $\delta_{i j}=5 \cdot 10^{2} / \mathrm{M} / \mathrm{s}$ in $(\mathrm{a})$, $\delta_{i j}=5 \cdot 10^{6} / \mathrm{M} / \mathrm{s}$ in (b), $\delta_{i j}=50 / \mathrm{M} / \mathrm{s}$ in (c); production rates $(k)$ are $2 \cdot 10^{3} / \mathrm{M} / \mathrm{s}$ in (a) and (c), $6 \cdot 10^{6}$ in (b). 